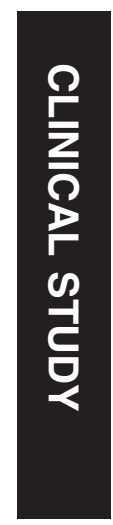

${ }^{1}$ Ophthalmology

Department,

Gloucestershire Eye Unit,

Cheltenham General

Hospital, Gloucestershire

Hospitals NHS Foundation

Trust, Gloucestershire, UK

${ }^{2}$ School of Engineering and

Physical Sciences,

Heriot-Watt University,

Edinburgh, UK

${ }^{3}$ School of Physics and Astronomy, University of

Glasgow, Glasgow, UK

${ }^{4}$ Cranfield University,

Bedfordshire, UK

Correspondence:

Al McNaught,

Ophthalmology Department, Gloucestershire Eye Unit,

Cheltenham General

Hospital, Gloucestershire

Hospitals NHS Foundation

Trust, Sandford Road,

Cheltenham, Gloucestershire

GL53 7AN, UK

Tel: +44 (0) 845422 5973;

Fax: +44 (0)1242 253816

Email: andy.mcnaught@

btopenworld.com

Received: 11 August 2013 Accepted in revised form: 28 April 2014

Published online: 25 July

2014

Previous presentations in meetings: The results of this manuscript were, in part, presented as a poster at the ARVO and Royal College of Ophthalmologists conferences in 2009

\section{Oxygen saturation measurements of the retinal vasculature in treated asymmetrical primary open-angle glaucoma using hyperspectral imaging}

\begin{abstract}
Purpose To determine whether there are differences in retinal vascular oxygen saturation measurements, estimated using a hyperspectral fundus camera, between normal eyes and treated eyes of subjects with asymmetrical primary open-angle glaucoma (POAG).

Methods A noninvasive hyperspectral fundus camera was used to acquire spectral images of the retina at wavelengths between 556 and $650 \mathrm{~nm}$ in 2-nm increments. In total, 14 normal eyes and both eyes of 11 treated POAG subjects were imaged and analyzed using algorithms that use the spectral variation of the optical densities of blood vessels to estimate the oxygen saturation of blood within the retinal vasculature. In the treated POAG group, each of the eyes were categorized, based on the mean deviation of the Humphrey visual-field analyzer result, as either more-advanced or less-advanced, glaucomatous eyes. Unpaired $t$-tests (twotailed) with Welch's correction were used to compare the mean oxygen saturation between the normal subjects and the treated POAG subgroups.

Results In less-advanced and moreadvanced-treated POAG eyes, mean retinal venular oxygen saturations $(48.2 \pm 21.6 \%$ and $42.6 \pm 18.8 \%$, respectively) were significantly higher than in normal eyes $(27.9 \pm 9.9 \%$; $P=0.03$ and 0.01 , respectively). Arteriolar oxygen saturation was not significantly different between normal eyes and treated POAG eyes.

Conclusions The increased oxygen saturation of the retinal venules in advancedtreated POAG eyes may indicate reduced metabolic consumption of oxygen in the inner retinal tissues.

Eye (2014) 28, 1190-1200; doi:10.1038/eye.2014.169; published online 25 July 2014

Introduction

It is currently acknowledged that in glaucoma, the inner retinal layer is the site of primary damage manifested by retinal ganglion cell (RGC) death. ${ }^{1}$ Raised intraocular pressure is the most important risk factor for glaucomatous RGC loss, but hypoxic stress, possibly as a result of blood-flow abnormalities, in the tissues of the optic nerve head and retina may also have a role in the pathogenesis of glaucoma. ${ }^{2-4}$ Regardless of the underlying mechanism of damage, measurements of tissue or, indirectly, intravascular oxygenation could be an informative method of determining the metabolic function of optic nerve and retina in glaucoma.

Measurements of oxygen tension overlying the optic nerve and retina have been reported in numerous animal studies; ${ }^{5-9}$ however, the technique involves the intraocular insertion of microelectrodes which is technically demanding and invasive. Phosphorescence imaging has been performed in experimental glaucoma in animals, ${ }^{10,11}$ but requires the injection of a bolus of an oxygen chemical probe whose safety in humans has yet to be determined.
\end{abstract}

DJ Mordant ${ }^{1}$, I Al-Abboud², G Muyo², A Gorman³, AR Harvey ${ }^{3}$ and Al McNaught ${ }^{1,4}$ 
Spectrophotometric techniques are currently the commonest noninvasive means of measuring the oxygen content of the retinal vasculature, retina, and optic nerve head. Studies using various retinal oximetry devices to measure the oxygen saturation of the retinal vasculature in glaucoma have been reported. ${ }^{12-20}$

Hyperspectral imaging combines spectroscopy with digital imaging whereby spectral data (using numerous contiguous wavelengths of light) are obtained in every pixel of an image plane. An oximetry study of the retinal vascular network using a hyperspectral imaging system (images acquired using more than three contiguous wavelengths) in glaucoma has not yet been reported. We have previously reported the validation of our hyperspectral oximetry technique using a model eye $\mathrm{e}^{21}$ and its application in normal subjects. ${ }^{22}$ The purpose of this study was to determine whether any differences in the oxygen saturation of the retinal vasculature between normal eyes and glaucomatous eyes could be detected using a hyperspectral imaging device.

\section{Materials and methods}

This study was conducted in accordance with the ethical standards of the Declaration of Helsinki, and was approved by the local Research and Ethics Committees at Gloucestershire Hospitals NHS Foundation Trust (REC reference number: 06/Q2005/132).

The hyperspectral fundus camera has been described previously. ${ }^{21,22}$ Briefly, it is a fundus camera incorporated with a liquid-crystal tunable filter in the optical path of the camera's light source, enabling the selection of wavelengths of illuminating light between 400 and $700 \mathrm{~nm}$ using a personal computer.

In total, 14 healthy subjects (some of whose data were published previously ${ }^{22}$ ) and 11 subjects with a diagnosis of asymmetrical POAG were recruited prospectively from the eye departments in Cheltenham and Gloucester General Hospitals between 2007 and 2010.

All subjects underwent full clinical ophthalmic evaluation, Snellen visual acuity testing, intraocular pressure measurement (IOP) using Goldmann applanation tonometry. The pupils were dilated with $1 \%$ tropicamide (Minims; Chauvin Pharmaceuticals, Romford, UK), and slit-lamp stereo biomicroscopy was performed to examine optic nerve head (at high magnification with a fundus $+78 \mathrm{D}$ lens) yielding the cup-to-disc ratio estimation, and detection of the qualitative indicators of glaucomatous damage. All POAG subjects had been tested with SITA Standard 24-2 perimetry (HFA II 740; Carl Zeiss Meditec, Jena, Germany).

Inclusion criteria for normal subjects were bestcorrected visual acuity (BCVA) of 6/9 or better, open anterior-chamber angle, absence of any ocular pathology,
IOP $<21 \mathrm{~mm} \mathrm{Hg}$, and normal optic disc appearance. Exclusion criteria included history of intraocular surgery, ocular pathology, diabetes mellitus, stroke, hypertension, and a family history of glaucoma. Inclusion criteria for POAG subjects included previously diagnosed open angles on gonioscopy and the presence of ocular hypertension (untreated IOP $>22 \mathrm{~mm} \mathrm{Hg}$ ), glaucomatous optic neuropathy (GON), and characteristic glaucomatous visual-field loss. Exclusion criteria included other previous or current ocular pathology and previous ocular surgery (except uncomplicated cataract surgery and trabeculectomy). Two subjects were current smokers and seven POAG subjects were treated with ocular anti-hypertensive therapy.

For the normal subjects, only one eye was selected randomly for analysis and for POAG subjects both eyes were analyzed. Each eye of the POAG subjects were divided into two categories, based on the mean deviation (MD) of the Humphrey visual-field analysis, with the more advanced having a more negative MD value than the less-advanced glaucomatous eye.

For each subject, a sequence of spectral retinal images between 556 and $650 \mathrm{~nm}$ in 2-nm increments were recorded. The processing and analysis of the spectral images have also been previously described in detail elsewhere. ${ }^{21,22}$ In brief, the optical density spectra (wavelengths from 556 to $650 \mathrm{~nm}$ ) of the tracked centreline points along the retinal vessels were calculated from the spectral images and applied to a non-linear oximetry model to yield an oxygen saturation estimate at each point. Wavelength-dependent optical scattering values published by Meinke et $a^{23}$ were used in this oximetry model. In essence, this algorithm estimates the free parameters based on the Levenberg-Marquardt nonlinear fit to OD $(\lambda)$ to yield an estimate of $c O S$.

For each eye, points along the $1^{\text {st }}$ degree superotemporal and infero-temporal vessels at the disc margin and $1 / 2$ disc diameter (dd) from the disc margin were located. The mean oxygen saturation at each of these points was calculated from 21 contiguous adjacent oxygen saturation measurements along each of the blood vessels.

The mean oxygen saturation at each location of the selected vessels was averaged to derive a single mean arteriolar and venular oxygen saturation of the normal eyes, POAG eyes with less-advanced glaucoma, and POAG eyes with more-advanced glaucoma.

\section{Statistical analysis}

The normality of the data distributions was tested using the D'Agostino and Pearson omnibus normality test, ${ }^{24}$ with the cut-off for non-normality set at $P<0.05$. All the data were confirmed to be normally distributed. 
Unpaired $t$-tests (two-tailed) with Welch's correction were used to compare the mean oxygen saturation of the temporal vessels between the normal subjects and the POAG subgroups. Paired $t$-tests (two-tailed) assuming unequal variance were used to compare the oxygen saturation of the temporal vessels between the eyes with more-advanced POAG and the respective contralateral eye with less-advanced POAG.

A post hoc power analysis using the mean oxygen saturation in each group, the average observed SD, and a 0.05 significance level were performed using a software program developed by Faul et al ${ }^{25}$ (Gpower program ver. 3.1).

All statistical analyses, unless otherwise indicated, were performed using statistical software (SPSS 19 for Windows; SPSS, Chicago, IL, USA); $P<0.05$ was considered statistically significant.

\section{Results}

Quantitative oximetry maps of the retinal vasculature and optical density spectra in a normal eye and an eye with advanced POAG

Pseudo-color images of the oxygen saturation values along the retinal vasculature in a normal eye and an eye with advanced glaucoma are illustrated in Figures 1 and 2 , respectively. The mean optical density spectra at selected segments ( $n=10$ spectra) of the 1st degree supero- and infero-temporal vessels are also illustrated to the right of each of the pseudo-color images. In both the normal and glaucomatous eye, the mean optical density spectra (red filled circles) and non-linear fit (red line) of selected locations along the first-degree supero- and infero-temporal arterioles show a plot with a trough and peak within the wavelength range of $560-590 \mathrm{~nm}$ (Figures 1a and 2c). This feature is consistent with the extinction coefficient of fully oxygenated hemoglobin (Figure 1c). ${ }^{23}$ The calculated oxygen saturations (derived from the mean optical density spectra) of the first-degree supero- and infero-temporal arterioles were 82.3 and $98.2 \%$ in the normal eye and 101.0 and $134.9 \%$ in the glaucomatous eye. In contrast, the mean optical density spectra (red filled circles) and non-linear fitting algorithms (red line) of selected locations along the firstdegree supero- and infero-temporal venules were different between the normal and glaucomatous eye (Figures $1 \mathrm{~b}$ and $2 \mathrm{~d}$ ). In the normal eye, the optical density spectra of the selected venules show a plot with a single peak at approximately $560 \mathrm{~nm}$ (Figure 1b). This feature is consistent with the extinction coefficient of deoxygenated hemoglobin (Figure 1c). ${ }^{23}$ The oxygen saturation calculations (from the mean optical density profile) were $26.1 \%$ and $27.8 \%$, respectively. In the glaucomatous eye, the mean optical density spectra of selected locations along the first-degree supero- and infero-temporal venules show a plot with a subtle trough between 570 and $580 \mathrm{~nm}$ (Figure 2d). The calculated oxygen saturations (from the mean optical density profile) were $39.2 \%$ and $41.7 \%$, respectively - higher than in the normal eye.

\section{Comparison of the oxygen saturation between normal subjects and patients with asymmetrical POAG}

The baseline characteristics of the study population are presented in Table 1. Compared with the normal subjects, treated POAG subjects had significantly worse BCVA, higher cup-to-disc ratio (vertical and horizontal), and higher systolic blood pressure.

The mean oxygen saturation of the temporal retinal arterioles and venules in normal subjects, and the treated POAG subgroups are summarized in Figure 3. In normal subjects, the mean oxygen saturation $( \pm S D)$ of the temporal retinal arterioles and venules were $104.82 \%$ $( \pm 18.67 \%)$ and $27.88 \%( \pm 9.89 \%)$, respectively. In the less-advanced and more-advanced glaucomatous eyes, the mean $( \pm S D)$ oxygen saturation of the temporal retinal arterioles were $98.10 \%( \pm 13.66 \%)$ and $106.00 \%$ $( \pm 22.00 \%)$, respectively. The mean $( \pm \mathrm{SD})$ oxygen saturation of the retinal venules were $48.19 \%( \pm 21.62 \%)$ and $42.60 \%$ ( $\pm 18.78 \%)$ in the less-advanced and moreadvanced glaucomatous eyes, respectively.

The mean oxygen saturation of the arterioles in normal subjects was not significantly different to that of the arterioles in the less- and more-advanced glaucomatous eyes $(P=0.31$ and 0.89 , respectively, unpaired $t$-test with Welch's correction). The mean oxygen saturation of the temporal retinal venules were significantly higher in both treated POAG subgroups compared with normal subjects $(P=0.03$ in eyes with less-advanced POAG and $P=0.01$ in eyes with more-advanced POAG, unpaired $t$-test with Welch's correction; post hoc power analysis: 0.82 and 0.65 , respectively).

Analysis of the two treated POAG subgroups (less- vs more-advanced visual-field loss) showed no significant difference in the mean arteriolar and venular oxygen saturation between the two groups $(P=0.33$ and 0.53 , respectively, paired $t$-test assuming unequal variance).

\section{Discussion}

The application of hyperspectral imaging to determine oxygen saturation differences in the retinal vasculature between normal and treated POAG subjects has been presented in this study. We have provided an illustration of the oxygen saturation calculations in detail at sample points along the retinal arterioles and venules in a 


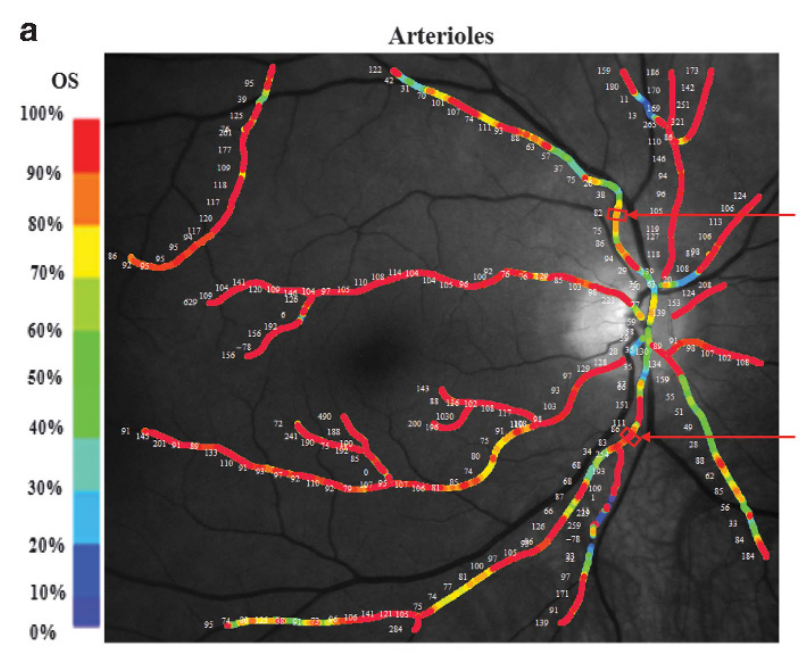

Mean optical density spectra, non-linear fitting oximetry algorithm and mean oxygen saturation calculation

at selected vessel segments
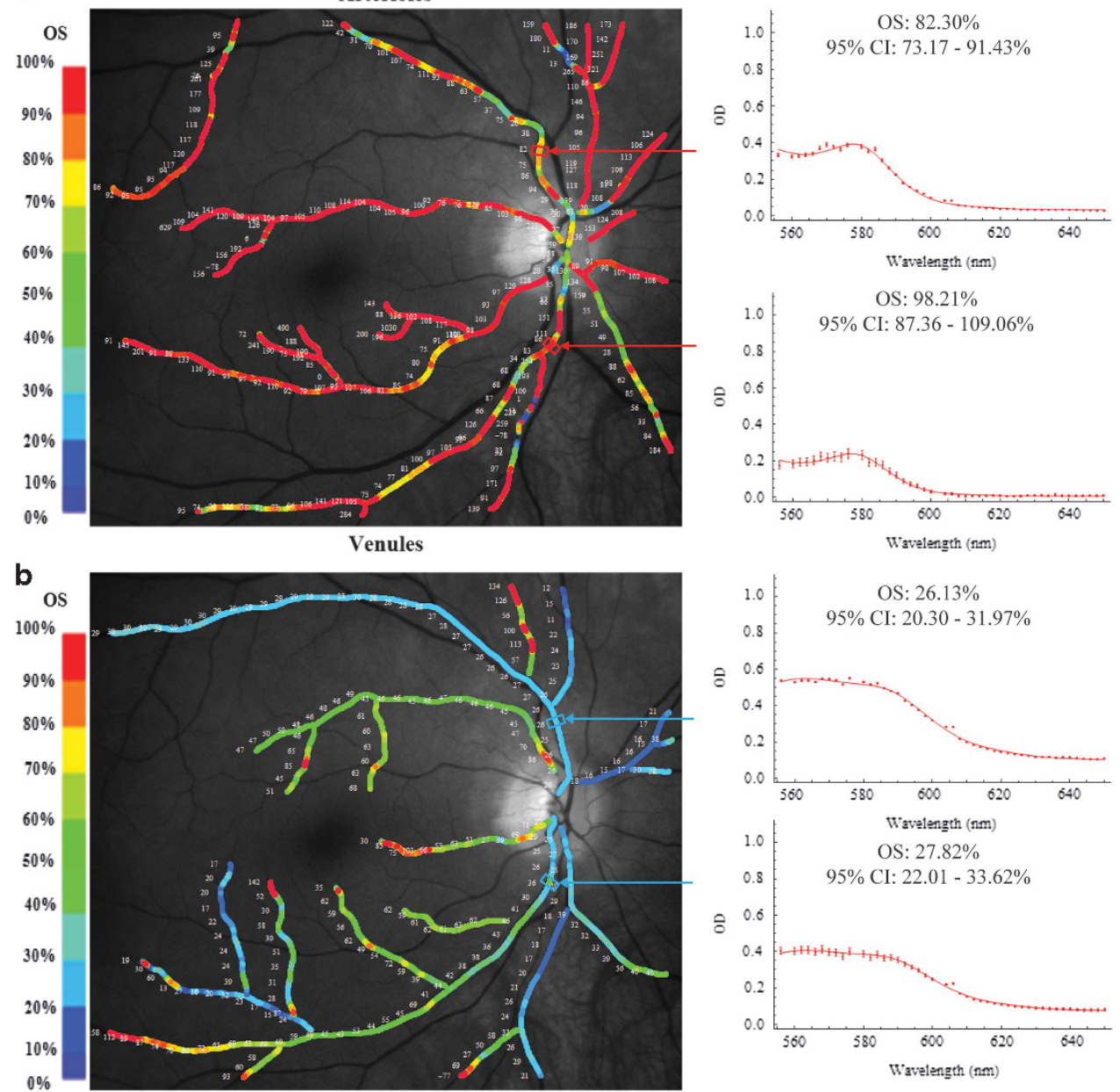

C $_{\text {Extinction coefficient spectra of hemoglobin in wavelengths between } 556 \text { and } 650 \mathrm{~nm}}$

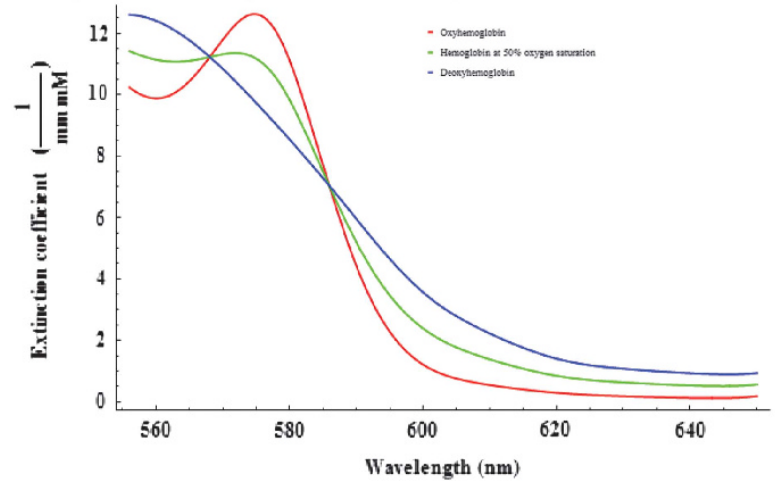

Figure 1 Pseudo-color images of the oxygen saturation values along the retinal arterioles ( $a$, left) and venules (b, left) in a normal eye. Mean optical density spectra (red filled circles and whiskers), non-linear fitting oximetry algorithm (red line), and oxygen saturation calculations of respective selected vessel segments are shown in the plots to the right. For comparison, the published extinction coefficient spectra of oxyhemoglobin, deoxyhemoglobin, and hemoglobin at 50\% oxygenation interpolated from van Assendelft's data are shown (c) ${ }^{23} \mathrm{CI}$, confidence interval; OS, oxygen saturation. 
a

Color fundus image

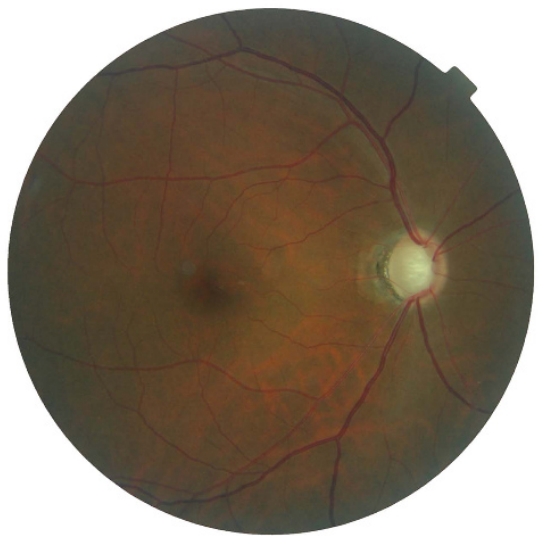

Pseudocolor quantitative oximetry maps of the retinal vasculature
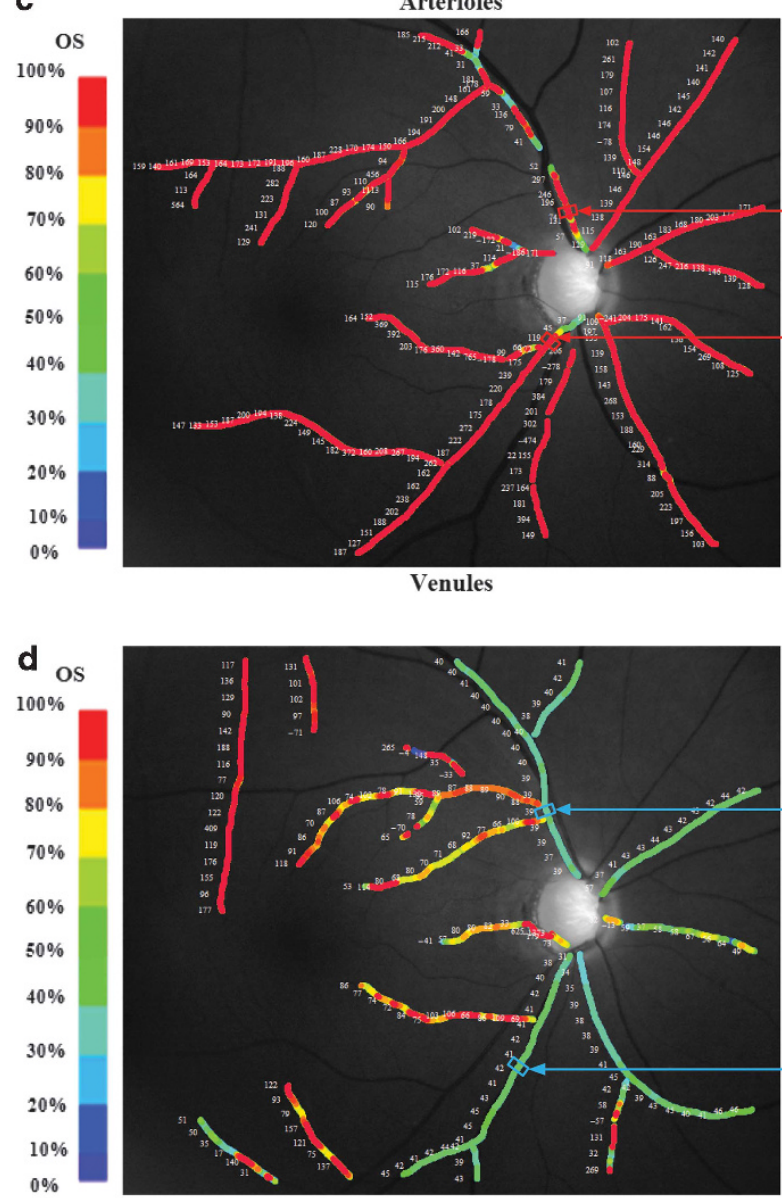

b

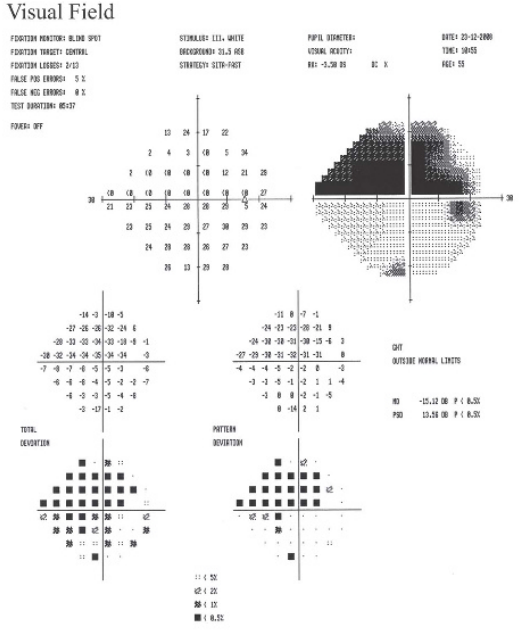

Mean optical density spectra, non-linear fitting oximetry algorithm and mean oxygen saturation calculation

at selected vessel segments
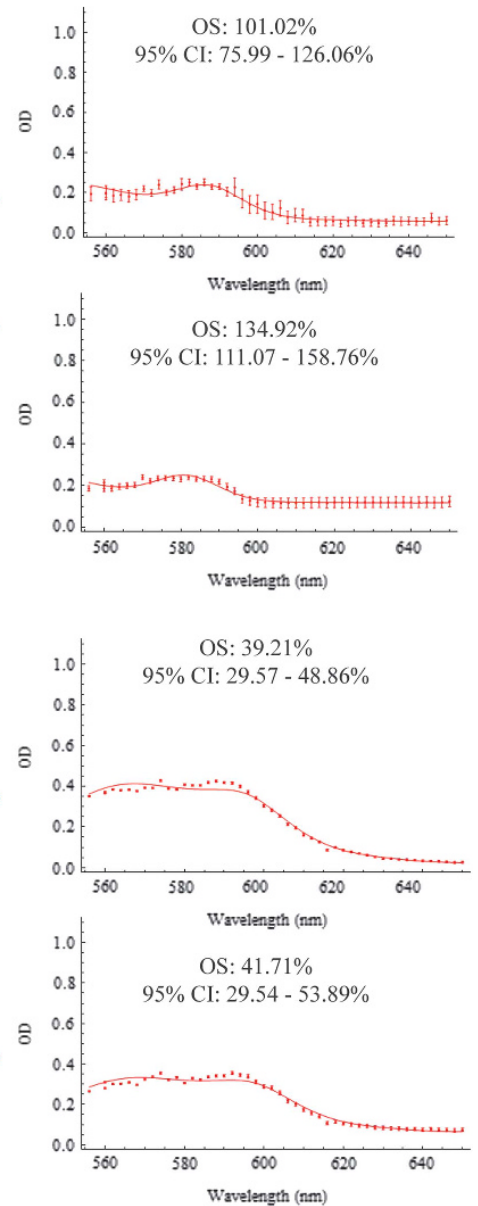

Figure 2 An eye with advanced glaucomatous optic neuropathy. A color fundus photograph showing advanced glaucomatous optic neuropathy (a) and Humphrey visual-field analysis showing a dense superior arcuate scotoma (b). Pseudo-color images of the oxygen saturation values along the retinal arterioles (c, left) and venules (d, left) in an eye with advanced POAG. Mean optical density spectra (red filled circles and whiskers), non-linear fitting oximetry algorithm (red line), and oxygen saturation calculations of respective selected vessel segments are shown in the plots to the right. CI, confidence interval; OS, oxygen saturation. 
Table 1 Baseline characteristics of the normal and treated POAG subjects included in the study

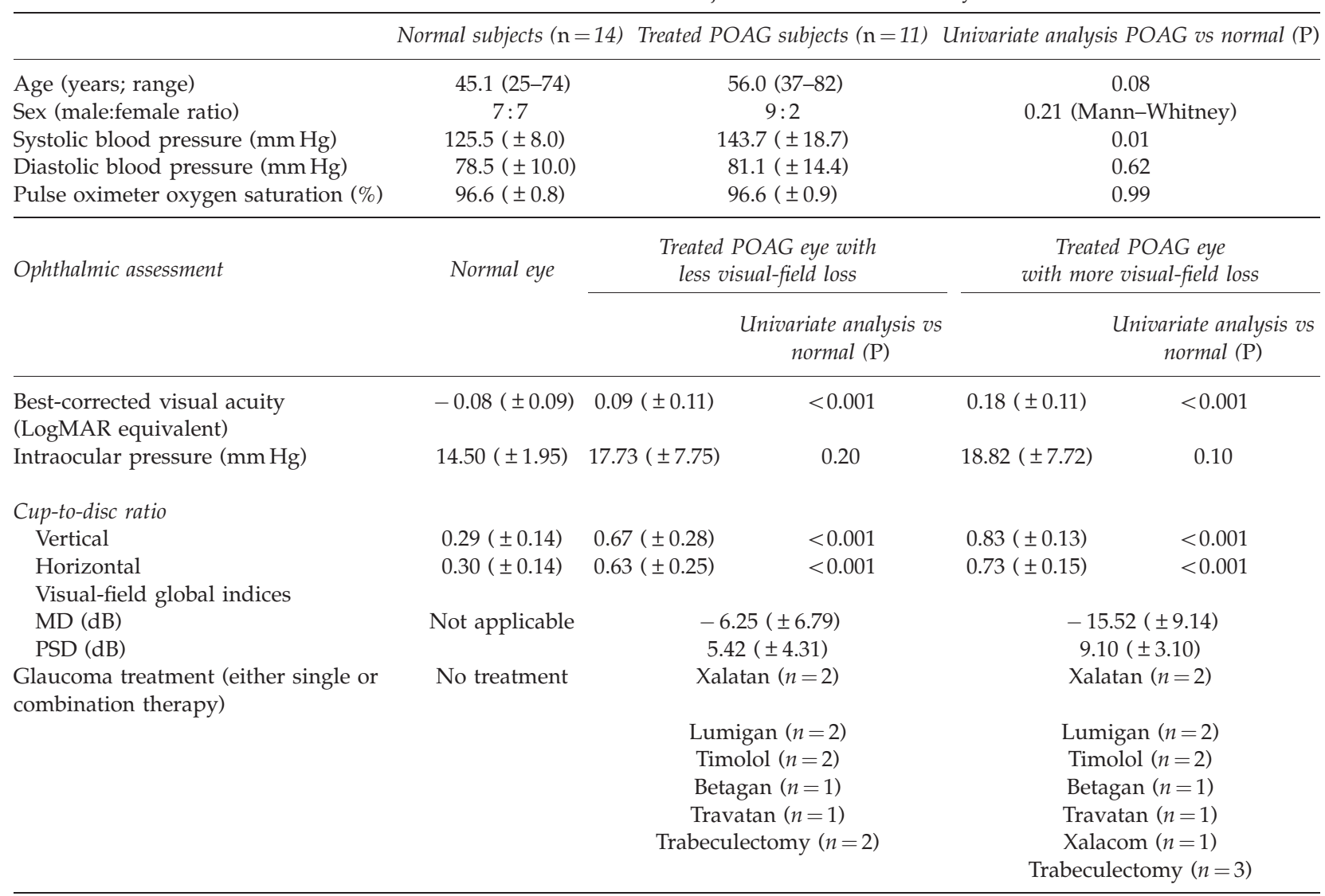

Data are presented as mean $( \pm \mathrm{SD})$ with univariate comparisons between normal and treated POAG subjects using unpaired $t$-test with Welch's correction unless stated otherwise. $\mathrm{Db}$, decibels; $\mathrm{MD}$, mean deviation; PSD, pattern standard deviation.

representative normal and glaucomatous eye. We have demonstrated differences in the mean optical density spectra at the sample points along the venules between the normal and glaucomatous eye which corresponds with detectable differences in the oxygen saturations. Our analysis of a cohort of normal and treated POAG subjects show significantly higher mean oxygen saturation of the temporal venules in POAG eyes compared with normal eyes. Arteriolar oxygen saturation was not significantly different between normal eyes and eyes with less- and more-advanced glaucomatous visual-field loss.

The higher mean oxygen saturation of the temporal retinal venules in glaucomatous eyes compared with normal eyes are contrary to a similar oximetry study, using a multi-wavelength imaging spectrometer, reported by Michelson and Scibor. ${ }^{17}$ In this study, no significant differences were observed in the oxygen saturation of the retinal venules between normal and glaucomatous eyes (normal-tension glaucoma and POAG). Arteriolar oxygen saturation was significantly lower in the normal-tension glaucoma cohort compared with normal subjects but not in the POAG cohort.
However, combining previous studies reported by Hardarson et al ${ }^{16,26}$ and Traustason et al, ${ }^{14}$ which used the same dual-wavelength retinal oximeter, the mean $( \pm S D)$ oxygen saturation of the retinal venules in glaucoma patients on topical medical therapy were $66 \%( \pm 5 \%)$ and $65 \%( \pm 6 \%)$, respectively. In glaucoma patients undergoing filtration surgery, the mean retinal venular oxygen saturation was $63 \%( \pm 5 \%)$ before surgery and $64 \%( \pm 6 \%)$ after surgery. ${ }^{16}$ Olafsdottir et al ${ }^{27}$ reported significantly higher retinal venular oxygen saturations using the same retinal oximeter in glaucomatous eyes with 'poor visual fields' compared with 'good visual fields', $68 \%( \pm 4 \%)$ and $62 \%( \pm 3 \%)$, respectively. Importantly, an earlier study by Hardarson et al ${ }^{16,27}$ found comparatively lower retinal mean $( \pm S D)$ venous oxygen saturation in normal subjects of 55\% ( $\pm 14 \%)$. A commonly accepted limitation of the two-wavelength oximetry technique is the tendency to overestimate the true blood oxygen saturation particularly at lower oxygen saturations. ${ }^{28}$ This could explain the higher venular oxygen saturation measurements in normal and glaucoma patients reported in these studies compared with our study. The relative difference in the venular 

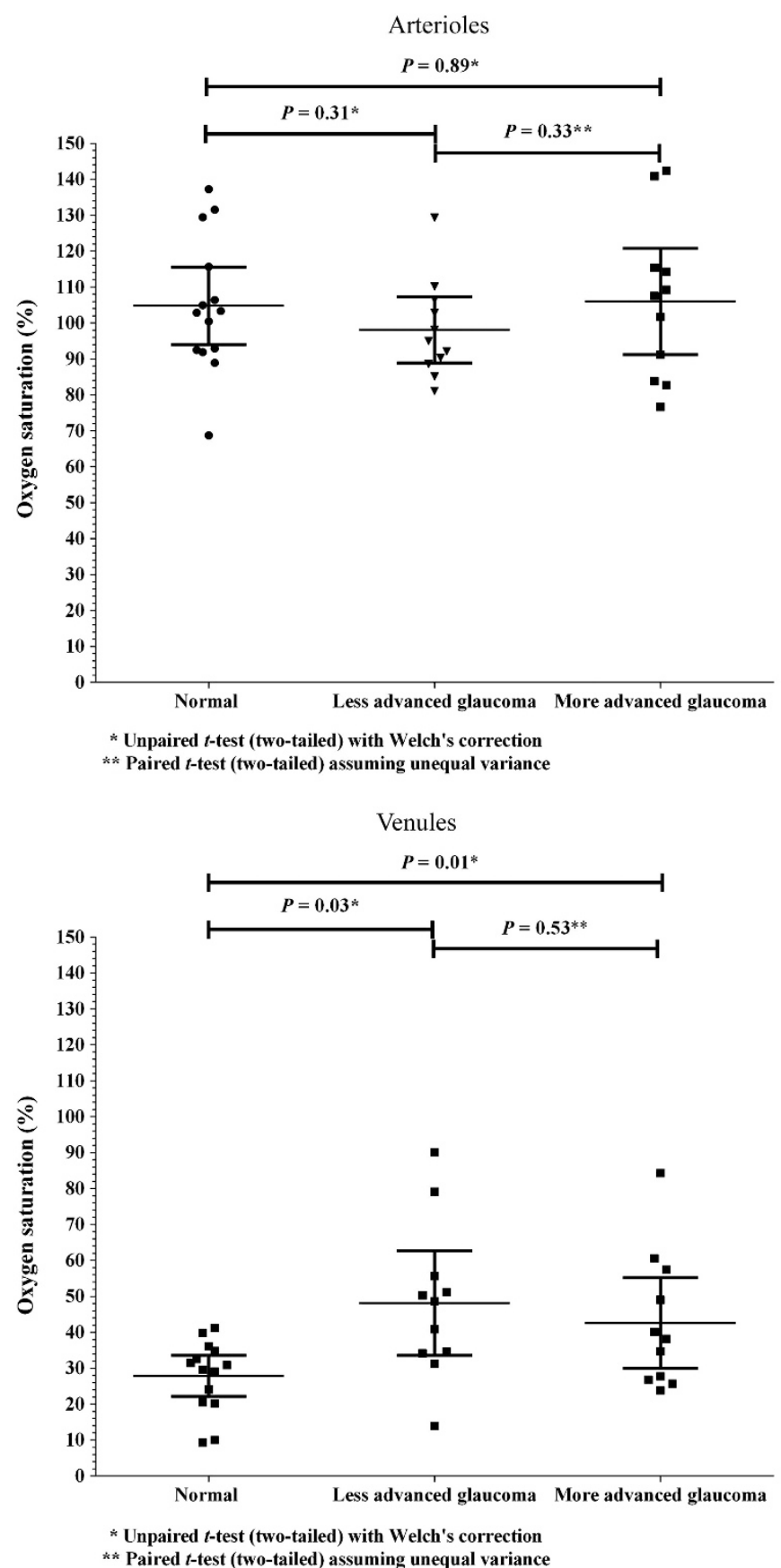

Summary of the oxygen saturation

\begin{tabular}{|c|c|c|c|c|c|c|}
\cline { 2 - 7 } \multicolumn{1}{c|}{} & \multicolumn{2}{c|}{ Normal subjects } & \multicolumn{2}{c|}{ Less advanced glaucomatous eyes } & \multicolumn{2}{c|}{ More advanced glaucomatous eyes } \\
\cline { 2 - 7 } \multicolumn{1}{c|}{} & $\begin{array}{c}\text { Arteriolar OS } \\
(\%)\end{array}$ & $\begin{array}{c}\text { Venular OS } \\
(\%)\end{array}$ & $\begin{array}{c}\text { Arteriolar OS } \\
(\%)\end{array}$ & $\begin{array}{c}\text { Venular OS } \\
(\%)\end{array}$ & $\begin{array}{c}\text { Arteriolar OS } \\
(\%)\end{array}$ & $\begin{array}{c}\text { Venular OS } \\
(\%)\end{array}$ \\
\hline Mcan & 104.82 & 27.88 & 98.10 & 48.19 & 106.00 & 42.60 \\
\hline SD & 18.67 & 9.89 & 13.66 & 21.62 & 22.00 & 18.78 \\
\hline $95 \% \mathrm{Cl}$ & $114.59-95.04$ & $33.05-22.70$ & $106.18-90.03$ & $60.97-35.41$ & $119.00-93.00$ & $53.70-31.51$ \\
\hline
\end{tabular}

Figure 3 A scatter plot of the oxygen saturation of the temporal retinal arterioles (top) and venules (middle) in the normal subjects and the POAG subgroups (less-advanced and more-advanced visual-field loss). Horizontal lines are the mean and 95\% confidence intervals. Mean oxygen saturation of the temporal retinal arterioles and venules in normal subjects and POAG subgroups are summarized in the table (bottom). CI, confidence interval, OS, oxygen saturation. 
oxygen saturation between normal subjects and glaucoma subjects (higher venular oxygen saturation in glaucoma patients), however, appears to support the findings in this study.

The increase in the oxygen saturation of the temporal retinal venules could indicate reduced metabolic consumption of oxygen in the retinal tissues in glaucomatous eyes. However, microcirculatory shunting of arterial blood into the venous system and physiological changes within the tissue causing a leftshift of the hemoglobin oxygen dissociation curve in the capillaries (increased affinity of hemoglobin for oxygen) cannot be excluded from our findings. Increased venous oxygen saturation is thought to be an indicator for reduced oxygen utilization as well as arteriovenous shunting. ${ }^{29}$ In normal human physiological conditions, the oxygen saturation of the veins draining their respective organs within the body is highly variable which is thought to reflect variations in the oxygen extraction levels or metabolic activity of the tissues. ${ }^{30}$ This is most evident in the renal venules whose oxygen saturation is approximately $92 \%$ as a result of relatively low-oxygen extraction in the renal tissues which derives its energy requirements from non-oxidative phosphorylation. ${ }^{31}$ In contrast, the oxygen saturation of venous drainage from the heart is approximately $37 \%$, which reflects high-oxygen extraction as a result of oxidative phosphorylation in the cardiac muscles. ${ }^{31}$ Rivers et $a l^{32}$ reported venous hyperoxia in patients following cardiac arrest, which was hypothesized to be a result of deranged systemic oxygen consumption.

In glaucoma, the reduced metabolic consumption of the retina could reflect neuronal loss and/or dysfunction. Numerous studies have reported the loss of ganglion cells in glaucoma ${ }^{33-40}$ and the underlying process is thought to involve apoptosis. ${ }^{41-44}$ Further, ganglion cell drop out is thought to be preceded by structural morphological changes of the dendrites ${ }^{45-47}$ and reduced sensitivity to light stimuli ${ }^{48,49}$ which suggests the presence of neuronal dysfunction which could be manifested by reduced oxygen consumption.

At an intracellular level, it has been postulated that mitochondrial function in the retinal ganglion cells are compromised by a multitude of insults which results in reduced energy production (reduced oxygen consumption) which subsequently induces cellular apoptosis. ${ }^{50}$ Ganglion cell loss and/or dysfunction on its own may not necessarily result in increased venous oxygen saturation, as other neuronal cells and supportive cells within the retinal layers require oxygen to fuel their metabolic demands. There is emerging evidence from histological, electrophysiological, and OCT studies that the processes involved in glaucomatous damage could also involve other neuronal cells such as amacrine cells, ${ }^{51}$ bipolar cells, ${ }^{50,52}$ horizontal cells, ${ }^{53}$ and photoreceptors. ${ }^{52,54-57}$ Therefore, reduced mass tissue extraction of oxygen within the retinal layers would support our explanation of retinal venous hyperoxia.

In this study, further analyses of the treated POAG subgroups revealed no significant differences in the oxygen saturation of the temporal retinal arterioles and venules between POAG eyes with less-advanced and more-advanced visual-field loss. In contrast, Vandewalle et $a l^{20}$ reported significantly higher venular oxygen saturation in patients with 'severe' visual-field defects compared with patients with 'mild' visual-field defects ( $69 \pm 3 \%$ vs $65 \pm 6 \%$, respectively; $P=0.0003$ ).

A major limitation of this study is the small sample size of patients included in this study and low statistical power (post hoc power analyses of all group comparisons, except between normal and lessadvanced POAG venules, were less than 0.8 ). The relatively few subjects imaged reflect the prolonged hyperspectral imaging routine required to yield the oximetry analysis: this is demanding for the imaged subject. In addition, the age difference between normal and POAG subjects approaches significance $(P=0.08)$. Therefore, caution should be used when interpreting the results of the study. Further studies would be useful to fully investigate the relationship between the oxygen saturation of the retinal vessels and glaucomatous damage with a larger population of matched subjects. Furthermore, in this study, our oxygen saturation calculations were performed on images captured in a single imaging session and therefore further studies on multiple measurements would be required to establish the extent or significance of short-term variations.

Other limitations from systematic errors such as a difference in the fundal reflectance between normal and glaucomatous eyes where there is a loss of neuronal tissue in the inner retina need to be considered. However, our previously reported model eye experiments demonstrated that background reflectance had no significant influence on the oxygen saturation measurements and did not affect its accuracy. ${ }^{21}$ Other physiological factors that could contribute toward a systematic error, such as blood flow and vessel diameter differences between normal subjects and glaucoma patients, are unlikely as these factors do not significantly change the characteristic shapes of the optical density profile that enable the calculation of the oxygen saturation. These physiological factors were previously studied by Schweitzer et al ${ }^{58}$ who demonstrated that in vitro changes to the blood flow and blood column thickness resulted in a vertical displacement of the spectral transmission profile of fully oxygenated blood without a significant change to the 
shape of the transmission profile. Therefore, the curve fitting technique used in this study to generate the oxygen saturation value is unlikely to be affected by such physiological features, as each oxygen saturation value is calculated from the specific shape of the optical density spectra. However, further research would be necessary to evaluate and confirm these statements.

In summary, we describe significant increases in the retinal venular oxygen saturation between normal and glaucomatous eyes using hyperspectral imaging. These findings may reflect reduced oxygen consumption by the impaired inner retina, and may therefore allow indirect assessments of retinal cell health using an imaging technology. If these findings are confirmed in a larger group of glaucoma patients, perhaps using the more clinically practical 'snapshot' hyperspectral imaging technology (which allows more rapid hyperspectral image collection), ${ }^{59}$ this imaging approach to the assessment of retinal function may prove useful in the diagnosis of inner retinal damage, and the monitoring of the response to treatment in glaucoma.

\section{Summary}

\section{What was known before}

- Retinal vascular oxygen saturation can be calculated using a variety of spectral imaging techniques. Most of these techniques involve dual-wavelength imaging.

- Higher venular oxygen saturation have been reported in glaucomatous eyes with 'poor visual fields' compared with glaucomatous eyes with 'good visual fields'.

What this study adds

- Retinal vascular oxygen saturation can be calculated using a hyperspectral imaging system.

- Venular oxygen saturation is higher in POAG eyes compared with normal eyes indicating reduced metabolic demand for oxygen.

\section{Conflict of interest}

ARH owns a patent to a related spectral imaging device, and the remaining authors declare no conflict of interest.

\section{Acknowledgements}

We thank the Gloucestershire Eye Therapy Trust and Technology Strategy Board (CHBT/007/00028). This paper presents independent research awarded under NEAT (New and Emerging Applications of Technology), part of the i4i Invention for Innovation programme (NEAT K034) of the National Institute for Health Research (NIHR).

\section{Disclaimer}

The views expressed in this publication are those of the author(s) and not necessarily those of the NHS, the NIHR or the Department of Health.

\section{References}

1 Quigley HA. Neuronal death in glaucoma. Prog Retin Eye Res 1999; 18(1): 39-57.

2 Yamazaki Y, Drance SM. The relationship between progression of visual field defects and retrobulbar circulation in patients with glaucoma. Am J Ophthalmol 1997; 124(3): 287-295.

3 Fontana L, Poinoosawmy D, Bunce CV, O'Brien C, Hitchings RA. Pulsatile ocular blood flow investigation in asymmetric normal tension glaucoma and normal subjects. Br J Ophthalmol 1998; 82(7): 731-736.

4 Sugiyama T, Schwartz B, Takamoto T, Azuma I. Evaluation of the circulation in the retina, peripapillary choroid and optic disk in normal-tension glaucoma. Ophthalmic Res 2000; 32(2-3): 79-86.

5 Ernest JT. In vivo measurement of optic-disk oxygen tension. Invest Ophthalmol Vis Sci 1973; 12(12): 927-931.

6 Ernest JT. Autoregulation of optic-disk oxygen tension. Invest Ophthalmol Vis Sci 1974; 13(2): 101-106.

7 Stefánsson E, Jensen PK, Eysteinsson T, Bang K, Kiilgaard JF, Dollerup J et al. Optic nerve oxygen tension in pigs and the effect of carbonic anhydrase inhibitors. Invest Ophthalmol Vis Sci 1999; 40(11): 2756-2761.

8 la Cour M, Kiilgaard JF, Eysteinsson T, Wiencke AK, Bang K, Dollerup J et al. Optic nerve oxygen tension: effects of intraocular pressure and dorzolamide. $\mathrm{Br} J$ Ophthalmol 2000; 84(9): 1045-1049.

9 Pedersen DB, Koch Jensen P, la Cour M, Kiilgaard JF, Eysteinsson T, Bang $\mathrm{K}$ et al. Carbonic anhydrase inhibition increases retinal oxygen tension and dilates retinal vessels. Graefes Arch Clin Exp Ophthalmol 2005; 243(2): 163-168.

10 Blumenröder S, Augustin AJ, Koch FH. The influence of intraocular pressure and systemic oxygen tension on the intravascular $\mathrm{pO} 2$ of the pig retina as measured with phosphorescence imaging. Surv Ophthalmol 1997; 42(Suppl 1): S118-S126.

11 Shonat RD, Wilson DF, Riva CE, Cranstoun SD. Effect of acute increases in intraocular pressure on intravascular optic nerve head oxygen tension in cats. Invest Ophthalmol Vis Sci 1992; 33(11): 3174-3180.

12 Khoobehi B, Beach JM, Kawano H. Hyperspectral imaging for measurement of oxygen saturation in the optic nerve head. Invest Ophthalmol Vis Sci 2004; 45(5): 1464-1472.

13 Siesky B, Harris A, Cantor LB, Kagemann L, Weitzman Y, McCranor L et al. A comparative study of the effects of brinzolamide and dorzolamide on retinal oxygen saturation and ocular microcirculation in patients with primary open-angle glaucoma. Br J Ophthalmol 2008; 92(4): 500-504.

14 Traustason S, Hardarson SH, Gottfredsdottir MS, Eysteinsson T, Karlsson RA, Stefánsson E et al. Dorzolamide-timolol combination and retinal vessel oxygen saturation in patients with glaucoma or ocular hypertension. Br J Ophthalmol 2009; 93(8): 1064-1067.

15 Siesky B, Harris A, Kagemann L, Stefansson E, McCranor L, Miller B et al. Ocular blood flow and oxygen delivery to the 
retina in primary open-angle glaucoma patients: the addition of dorzolamide to timolol monotherapy. Acta Ophthalmol 2010; 88(1): 142-149.

16 Hardarson SH, Gottfredsdottir MS, Halldorsson GH, Karlsson RA, Benediktsson JA, Eysteinsson T et al. Glaucoma filtration surgery and retinal oxygen saturation. Invest Ophthalmol Vis Sci 2009; 50(11): 5247-5250.

17 Michelson G, Scibor M. Intravascular oxygen saturation in retinal vessels in normal subjects and open-angle glaucoma subjects. Acta Ophthalmol Scand 2006; 84: 89-295.

18 Ito M, Murayama K, Deguchi T, Takasu M, Gil T, Araie M et al. Oxygen saturation levels in the juxta-papillary retina in eyes with glaucoma. Exp Eye Res 2008; 86(3): 512-518.

19 Schweitzer D, Guenther S, Scibor M, Hammer M. Spectrometric investigations in ocular hypertension and early stages of primary open angle glaucoma and of low tension glaucoma-multisubstance analysis. Int Ophthalmol 1992; 16(4-5): 251-257.

20 Vandewalle E, Abegão Pinto L, Olafsdottir OB, De Clerck E, Stalmans P, Van Calster J et al. Oximetry in glaucoma: correlation of metabolic change with structural and functional damage. Acta Ophthalmol 2013; 92(2): 105-110.

21 Mordant DJ, Al-Abboud I, Muyo G, Gorman A, Sallam A, Rodmell $\mathrm{P}$ et al. Validation of human whole blood oximetry, using a hyperspectral fundus camera with a model eye. Invest Ophthalmol Vis Sci 2011; 52(5): 2851-2859.

22 Mordant DJ, Al-Abboud I, Muyo G, Gorman A, Sallam A, Ritchie P et al. Spectral imaging of the retina. Eye 2011; 25(3): 309-320.

23 Meinke M, Müller G, Helfmann J, Friebel M. Empirical model functions to calculate hematocrit-dependent optical properties of human blood. Appl Opt 2007; 46(10): 1742-1753.

24 D'Agostino RB, Belanger A, D'Agostino Jr RB. A suggestion for using powerful and informative tests of normality. Am Statistician 1990; 13(4): 316-321.

25 Faul F, Erdfelder E, Buchner A, Lang AG. Statistical power analyses using $G^{*}$ Power 3.1: tests for correlation and regression analyses. Behav Res Methods 2009; 41: 1149-1160.

26 Hardarson SH, Harris A, Karlsson RA, Halldorsson GH, Kagemann L, Rechtman E et al. Automatic retinal oximetry. Invest Ophthalmol Vis Sci 2006; 47(11): 5011-5016.

27 Olafsdottir OB, Hardarson SH, Gottfredsdottir MS, Harris A, Stefánsson E. Retinal oximetry in primary open-angle glaucoma. Invest Ophthalmol Vis Sci 2011; 52(9): 6409-6413.

28 Sarnquist F, Todd C, Whitcher C. Accuracy of a new non-invasive oxygen saturation monitor. Anesthesiology 1980; 53: S163.

29 Marx G, Reinhart K. Venous oximetry. Curr Opin Crit Care 2006; 12(3): 263-268.

30 Reinhart K. Monitoring O2 transport and tissue oxygenation in critically ill patients. In: Reinhart K, Eyrich $\mathrm{K}$ eds. Clinical Aspects of O2 Transport and Tissue Oxygenation. Springer: Berlin, Heidelberg, 1989.

31 Byrick RJ, Rose DK. Pathophysiology and prevention of acute renal failure: the role of the anaesthetist. Can J Anaesth 1990; 37(4 Pt 1): 457-467.

32 Rivers EP, Rady MY, Martin GB, Fenn NM, Smithline HA, Alexander ME et al. Venous hyperoxia after cardiac arrest. Characterization of a defect in systemic oxygen utilization. Chest 1992; 102: 1787-1793.

33 Quigley HA, Addicks EM. Chronic experimental glaucoma in primates, II: effect of extended intraocular pressure elevation on optic nerve head and axonal transport. Invest Ophthalmol Vis Sci 1981; 19: 137-152.

34 Quigley HA, Addicks EM, Green WR, Maumenee AE. Optic nerve damage in human glaucoma. II. The site of injury and susceptibility to damage. Arch Ophthalmol 1981; 99: 635-649.

35 Morgan JE, Uchida H, Caprioli J. Retinal ganglion cell death in experimental glaucoma. Br J Ophthalmol 2000; 84: 303-310.

36 Glovinsky Y, Quigley HA, Dunkelberger GR. Retinal ganglion cell loss is size dependent in experimental glaucoma. Invest Ophthalmol Vis Sci 1991; 32: 484-491.

37 Glovinsky Y, Quigley HA, Pease ME. Foveal ganglion cell loss is size dependent in experimental glaucoma. Invest Ophthalmol Vis Sci 1993; 34: 395-400.

38 Weber AJ, Kaufman PL, Hubbard WC. Morphology of single ganglion cells in the glaucomatous primate retina. Invest Ophthalmol Vis Sci 1998; 39: 2304-2320.

39 Hitchings RA. Selective ganglion cell death in glaucoma. Br J Ophthalmol 2000; 84: 678-679.

40 Quigley HA, Sanchez RM, Dunkelberger GR, L'Hernault NL, Baginski TA. Chronic glaucoma selectively damages large optic nerve fibers. Invest Ophthalmol Vis Sci 1987; 28: 913-920.

41 Kerrigan LA, Zack DJ, Quigley HA, Smith SD, Pease ME. TUNEL-positive ganglion cells in human primary openangle glaucoma. Arch Ophthalmol 1997; 115(8): 1031-1035.

42 Quigley HA, Nickells RW, Kerrigan LA, Pease ME, Thibault DJ, Zack DJ. Retinal ganglion cell death in experimental glaucoma and after axotomy occurs by apoptosis. Invest Ophthalmol Vis Sci 1995; 36: 774-786.

43 Garcia-Valenzuela E, Shareef S, Walsh J, Sharma SC. Programmed cell death of retinal ganglion cells during experimental glaucoma. Exp Eye Res 1995; 61(1): 33-44.

44 Guo L, Moss SE, Alexander RA, Ali RR, Fitzke FW, Cordeiro MF. Retinal ganglion cell apoptosis in glaucoma is related to intraocular pressure and IOP-induced effects on extracellular matrix. Invest Ophthalmol Vis Sci 2005; 46(1): 175-182.

45 Morgan JE, Datta AV, Erichsen JT, Albon J, Boulton ME. Retinal ganglion cell remodelling in experimental glaucoma. Adv Exp Med Biol 2006; 572: 397-402.

46 Shou T, Liu J, Wang W, Zhou Y, Zhao K. Differential dendritic shrinkage of alpha and beta retinal ganglion cells in cats with chronic glaucoma. Invest Ophthalmol Vis Sci 2003; 44: 3005-3010.

47 Weber AJ, Kaufman PL, Hubbard WC. Morphology of single ganglion cells in the glaucomatous primate. Invest Ophthalmol Vis Sci 1998; 39: 2304-2320.

48 Porciatti V. Pattern electroretinogram in glaucoma. Curr Opin Ophthalmol 2006; 17(2): 196-202.

49 North RV, Jones AL, Drasdo N, Wild JM, Morgan JE. Electrophysiological evidence of early functional damage in glaucoma and ocular hypertension. Invest Ophthalmol Vis Sci 2010; 51(2): 1216-1222.

50 Osborne NN. Pathogenesis of ganglion "cell death" in glaucoma and neuroprotection: focus on ganglion cell axonal mitochondria. Prog Brain Res 2008; 173: 339-352.

51 Raz D, Perlman I, Percicot CL, Lambrou GN, Ofri R. Functional damage to inner and outer retinal cells in experimental glaucoma. Invest Ophthalmol Vis Sci 2003; 44(8): 3675-3684.

52 Lei Y, Garrahan N, Hermann B, Becker DL, Hernandez MR, Boulton ME et al. Quantification of retinal transneuronal degeneration in human glaucoma: a novel multiphotonDAPI approach. Invest Ophthalmol Vis Sci 2008; 49: 1940-1945. 
53 Janssen P, Naskar R, Moore S, Thanos S, Thiel H. Evidence for glaucoma-induced horizontal cell alterations in the human retina. Ger J Ophthalmol 1996; 5: 378-385.

54 Nork TM, Ver Hoeve JN, Poulsen GL, Nickells RW, Davis MD, Weber AJ et al. Swelling and loss of photoreceptors in chronic human and experimental glaucomas. Arch Ophthalmol 2000; 118: 235-245.

55 Panda S, Jonas JB. Decreased photoreceptor count in human eyes with secondary angle-closure glaucoma. Invest Ophthalmol Vis Sci 1992; 33: 2532-2536.

56 Velten IM, Korth M, Horn FK. The a-wave of the dark adapted electroretinogram in glaucomas: are photoreceptors affected? Br J Ophthalmol 2001; 85: 397-402
57 Guo L, Normando EM, Nizari S, Lara D, Cordeiro MF Tracking longitudinal retinal changes in experimental ocular hypertension using the cSLO and spectral domain-OCT. Invest Ophthalmol Vis Sci 2010; 51(12): 6504-6513.

58 Schweitzer D, Hammer M, Kraft J, Thamm E, Königsdörffer E, Strobel J. In vivo measurement of the oxygen saturation of retinal vessels in healthy volunteers. IEEE Trans Biomed Eng 1999; 46(12): 1454-1465.

59 Gorman A, Fletcher-Holmes DW, Harvey AR. Generalization of the Lyot filter and its application to snapshot spectral imaging. Opt Express 2010; 18(6): 5602-5608. 